\title{
Precise Microfluidic Luminescent Sensor Platform with Controlled Injection System
}

\author{
Bongsu Kang," Sunghak Choi," Keesung Kim, Ho-Sup Jung,* and Moon Kyu Kwak*
}

Cite This: ACS Omega 2021, 6, 23412-23420

Read Online

ABSTRACT: Efforts have been devoted to screening various prevalent diseases, such as severe acute respiratory syndrome (SARS) and coronavirus disease 2019 (COVID-19). Real-time polymerase chain reaction (RT-PCR), which is currently the most widely used, has high accuracy, but it requires several facilities and takes a relatively long time to check; so, new testing technology is necessary for a higher test efficiency. A chemiluminescence (CL) sensor is a relatively simple device and suitable as an alternative because it can detect very precise specimens. However, in measurements via CL, the quantitative formulation of reagents that cause color development is important. In the case of mixing using micropipettes, precise analysis is possible, but this technique is limited by uncontrollable errors or deviations in detection amounts. In addition, in using a microfluidic chip to increase field applicability, a syringe pump or other quantification injection tools are required, so problems must be overcome for practical use. Therefore, in this study, a microchip was designed and manufactured to supply a sample of a certain volume by
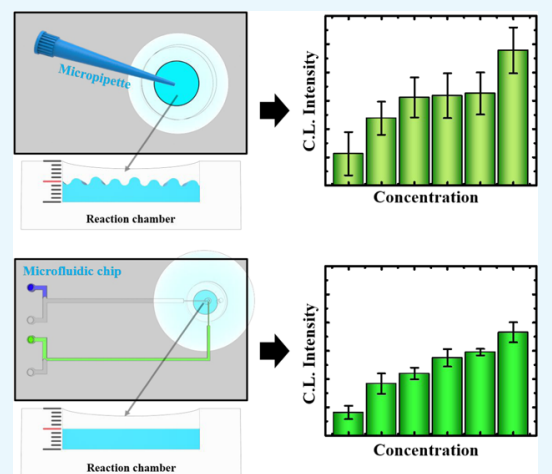
simply blowing air and injecting a sample into the chamber. By utilizing the luminescence reaction of luminol, $\mathrm{CuSO}_{4}$ and $\mathrm{H}_{2} \mathrm{O}_{2}$ the performance of the prepared chip was confirmed, and the desired amount of the sample could be injected with a simple device with an error rate of $2 \%$ or less. For feasible applications, an experiment was performed to quantitatively analyze thrombin, a biomarker of heart disease. Results demonstrated that biomarkers could be more precisely detected using the proposed microchips than using micropipettes.

\section{INTRODUCTION}

Global pandemic diseases, namely, severe acute respiratory syndrome (SARS) and coronavirus disease 2019 (COVID-19), have posed a severe risk on human populations, with increasing infection rates. Moreover, we predict the occurrence of highrisk infection diseases with unknown viruses and pathogens earlier in the future. With the emphasis of studies on infections and diseases shifting toward prevention, early detection, and monitoring, hassle-free and patient-centered sensor technologies, such as technologies of point-of-care (POC) testing, should be developed.

POC diagnostic devices are inexpensive, easy to use, rapid, and instrument-independent. An idealized POC device can operate with small sample volumes of complex biological and chemical samples. As a result, POC diagnostics can reduce medical costs, mislabeling, mishandling, and analysis misdirection. ${ }^{1-3}$ Results can be obtained more quickly, enabling more effective treatments of progressing diseases and infection outbreaks. As such, POC devices are used for the prevention and control of disease outbreaks and the monitoring of health conditions in remote areas and nonlaboratory settings, where a lack of professional technicians and sophisticated laboratory infrastructures reduces healthcare parameters. Recent advances in microfluidic integration, smart soft materials, data analytics, and connectivity have improved the performance of POC diagnostic devices. ${ }^{4,5}$ For instance, microfluidic platforms have been developed to enhance the analysis and synthesis of materials by precisely controlling small volumes of sample solution according to their purpose and application to other disciplines since the concept of lab-on-chip (LOC) was conceived in $1979 .^{6-8}$ This LOC platform has been intensively investigated and miniaturized with the discovery and development of photolithography ${ }^{9,10}$ and soft lithography, ${ }^{11,12}$ which are used to fabricate nano/microstructures. ${ }^{13,14}$ LOC is a tool applied to various laboratory-scale experiments composed of microfluidic channels, which are smaller than a palm. Moreover, the LOC platform can be expanded to POC diagnostic sensor devices composed of a reservoir, microfluidic channels, mixing structures, and a signal detection area. Numerous POC diagnostic sensors based on LOC technologies have been constructed using optical, electrochemical, and surface plasmon resonance (SPR) techniques and chemical luminescence $(\mathrm{CL}) .^{15,16}$

Received: June 26, 2021

Accepted: August 18, 2021

Published: September 2, 2021 
(a)

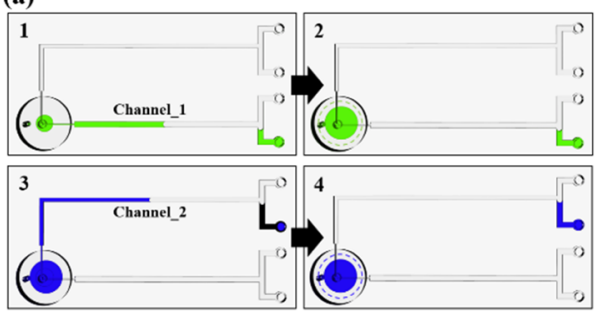

(b)

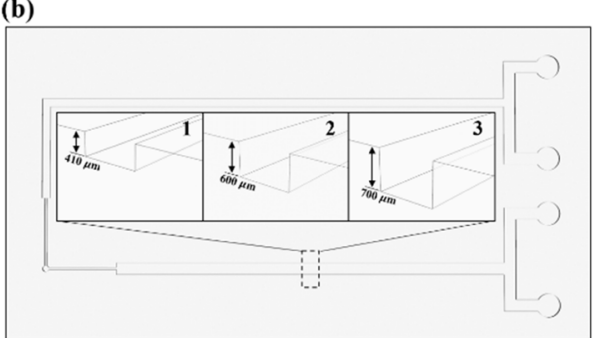

(c)

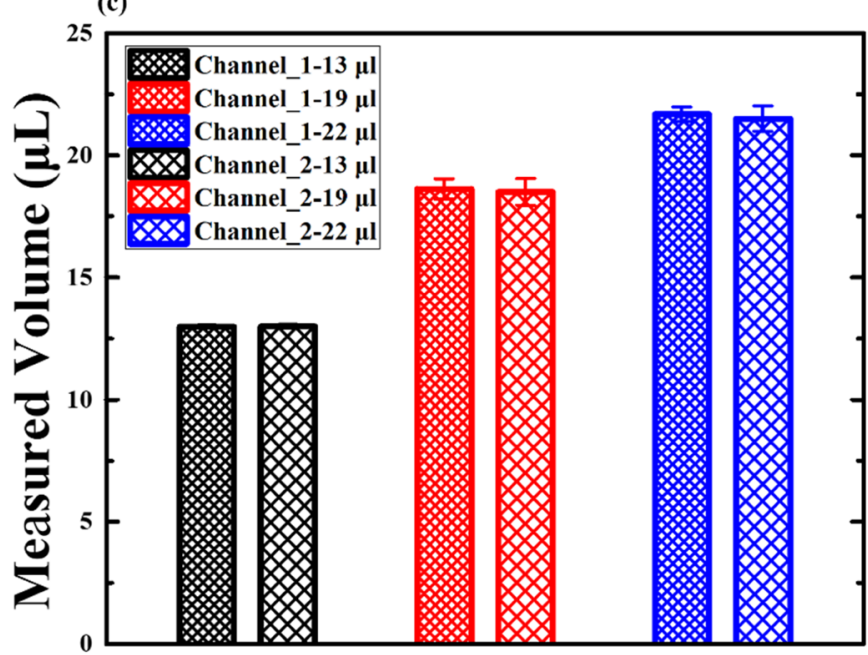

Figure 1. Control of quantitative injection sample reagents through two channels by injection of air. (a) First reagent of channel 1 moves to the reaction chamber $(1 \rightarrow 2)$, and the second reagent of channel 2 moves to the reaction chamber $(3 \rightarrow 4)$ by air on another port. (b) Heights of the channels are 410,600, and $700 \mu \mathrm{m}$, and their target volumes are 13,19 , and $22 \mu \mathrm{L}$, respectively. (c) Measurement of each reaction chamber volume depending on the height of the microfluidic chip channel. Channels 1 and 2: target (black): $12.99 \pm 0.07,13.0 \pm 0.07 \mu \mathrm{L}$; target (red): $18.62 \pm$ $0.4118 .5 \pm 0.54 \mu \mathrm{L}$; target (blue): $21.68 \pm 0.3,21.5 \pm 0.51 \mu \mathrm{L}$.

(a)

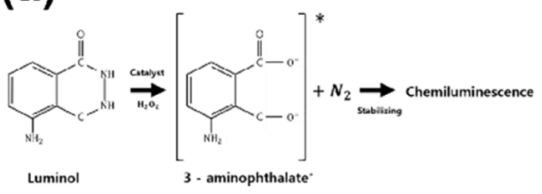

(b)
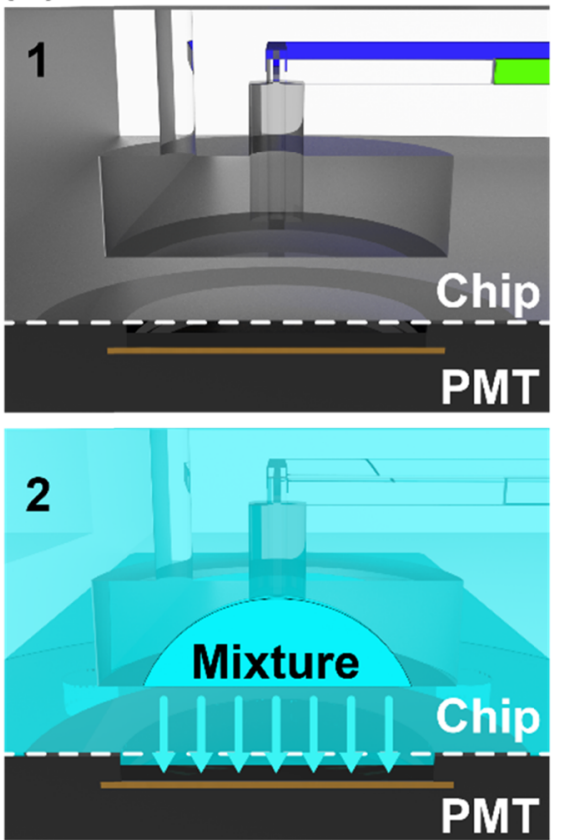

(c)
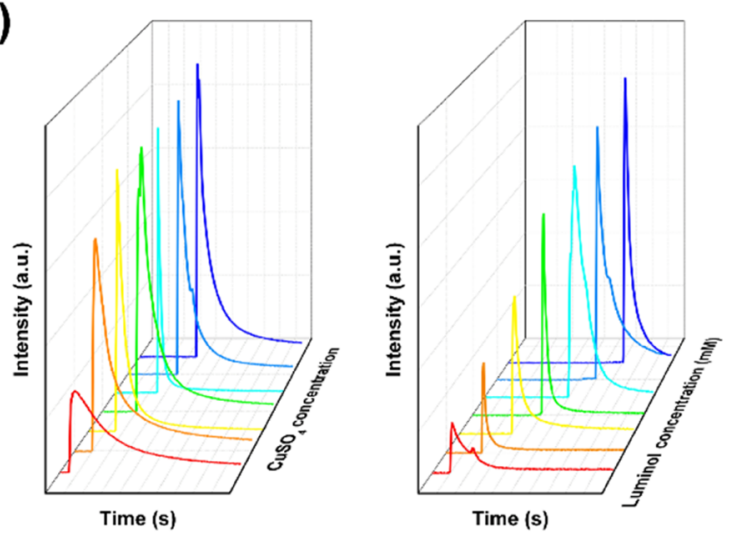

Time (s)

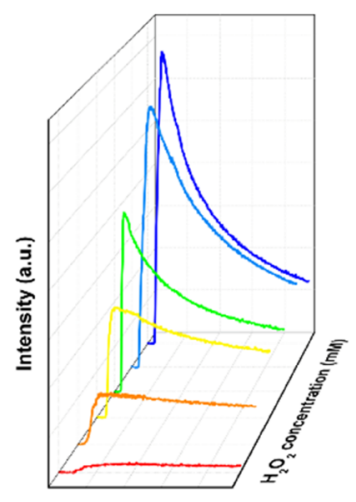

Time (s)

(d)

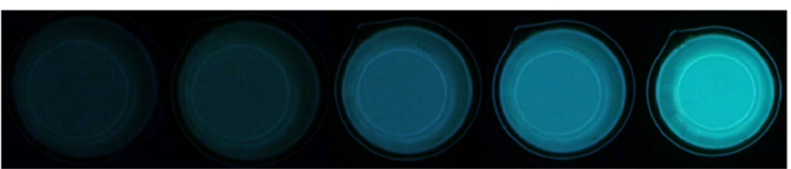

(e)
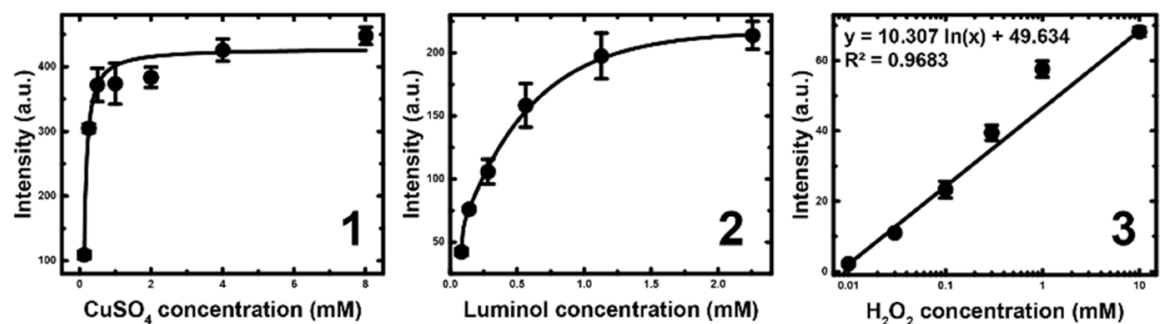

Figure 2. (a) Luminol CL exhibits an efficient catalytic activity of luminol in alkaline solution in the presence of $\mathrm{CuSO}_{4}$ and $\mathrm{H}_{2} \mathrm{O}_{2}$. (b) Schematic of the injection process (from 1 to 2): for $\mathrm{CL}$ intensity generation, reagent 1 containing luminol and $\mathrm{CuSO}_{4}$ is injected into the reaction chamber through channel 1, and reagent 2 containing $\mathrm{H}_{2} \mathrm{O}_{2}$ is carried at the reaction chamber through channel 2. (c) CL intensity-time curves according to luminol, $\mathrm{CuSO}_{4}$, and $\mathrm{H}_{2} \mathrm{O}_{2}$ concentration. (d) $\mathrm{CL}$ emission in luminol $\mathrm{CL}$ with increasing $\mathrm{H}_{2} \mathrm{O}_{2}$ concentrations from left to right. (e) CL intensity-concentration curves of luminol $\left(1 \mathrm{mM} \mathrm{H}_{2} \mathrm{O}_{2}, 1 \mathrm{mM} \mathrm{CuSO}_{4}\right), \mathrm{CuSO}_{4}\left(3 \mathrm{mM}\right.$ luminol, $\left.1 \mathrm{mM} \mathrm{H}_{2} \mathrm{O}_{2}\right)$, and $\mathrm{H}_{2} \mathrm{O}_{2}(0.1 \mathrm{mM} \mathrm{luminol}, 1 \mathrm{mM}$ $\left.\mathrm{CuSO}_{4}\right)$. 


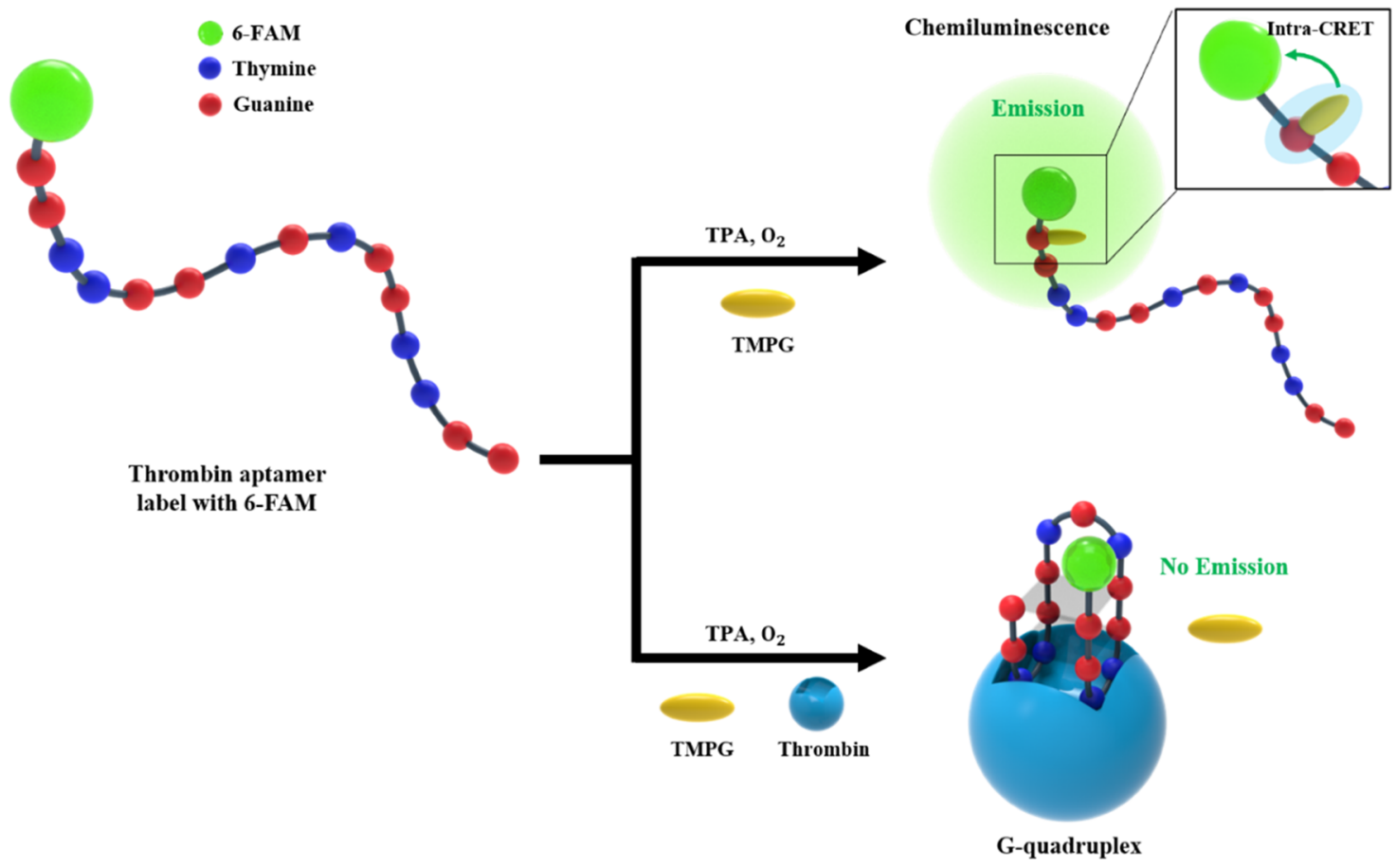

Figure 3. Schematic of the CL generation mechanism based on the thrombin aptamer (TBA) conjugated with 6-FAM. TMPG binds to guanine of the aptamer and transfers intra-CRET to 6-FAM, which intensifies the generated strong CL. The TBA-thrombin complex decreases the dim light produced by the G-quadruplex structure, which has no site to bind to TMPG.

CL sensors have been used to detect the concentration and content of target materials by evaluating the luminescent reaction generated from a mixture of a luminescent reagent and a target sample. ${ }^{17,18} \mathrm{CL}$ studies have been performed to develop an accurate and rapid analysis method that uses only a small amount of reagents; the detection precision of this method is higher than that of other similar optical analysis techniques, such as adsorption UV spectrometer and fluorescence analyses. ${ }^{19}$ Indeed, the CL method is applicable to various detection and analysis fields, such as gas and liquid component analysis, organic matter analysis, DNA sequencing, and combustion analysis. ${ }^{20-23}$ For typical applications, however, CL detection requires specific quantitative conditions of reagents and samples, ${ }^{24,25}$ expensive analytical equipment, and specific reagents. Even if this method provides precise and accurate analytical data, typical CL is not user-friendly and has low accessibility, so it is only utilized in laboratories with related complex equipment.

In this study, a CL detection device with a quantification injection function was developed by using microchannels to achieve effortless sample quantification and easy sensing. The significance of this study is that a novel microfluidic chip system with a precise amount of reagents and small equipment was designed for the CL detection of luminol and thrombin as a model target sample by controlling the microfluids. However, the application of microfluidic chips for miniaturizing and simplifying the scale of CL experiments has not been universal.

\section{RESULTS AND DISCUSSION}

2.1. Quantitative Controlled Injection Volume Depends on Channel Geometry. Luminescence intensity measurements are sensitive to variations in the illumination pathway, including the light source itself, interfering ambient light, and variations in detectors. The quantification of analytes can also be affected by variations in the concentrations of dyes in matrices, photobleaching, and light scattering. Therefore, the amount of CL reagents needs to be precisely controlled to detect a target material quantitatively. In this study, a microfluidic chip was designed to control the injection volume and maintain the concentration of CL reagent without using other devices, such as syringe pumps and micropipettes. Indeed, our microfluidic chip could hold a minimum volume of expensive reagents for detection. First, both channels are filled with the sample reagents from one port and an appropriate amount of air is blown through another port to reach the reaction chamber. Second, the reagent of channel 2 is similarly moved into the reaction chamber (Figure 1a). Moreover, we verified to control of injection volume by channels which are fabricated three different height and same wide, quantitively. The heights of the channels are 410,600, and $700 \mu \mathrm{m}$ (Figure $1 b)$, and their target volumes are 13, 19, and $22 \mu \mathrm{L}$, respectively. Experiments were replicated 40 times for each injection volume depending on the height of channels. Figure 1c shows that the measurement of reaction chamber volume almost reached the target volume, and no significant variation was observed under $\pm 1 \mu \mathrm{L}$ even though the blown air volume was not exact.

As a result, the fabricated microchip could precisely control the injection volume and quantitatively analyze the volume of the injected specimen through a CL technique. Especially, when a micropipette was used to mix the reagents, the CL intensity varied because it is difficult to inject the precise amount of reagent using micropipettes. Several problems occur, such as the quantitative detection of target samples, waste of reagents, and loss of CL intensity during processing. Our microfluidic chip could exactly control the volume of reagents depending on the height or width of channels by blowing an appropriate amount of air without any fine injection devices. 
(a)

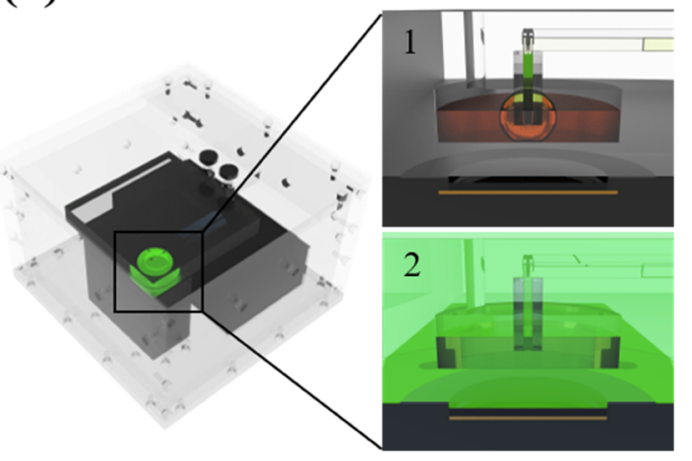

(c)

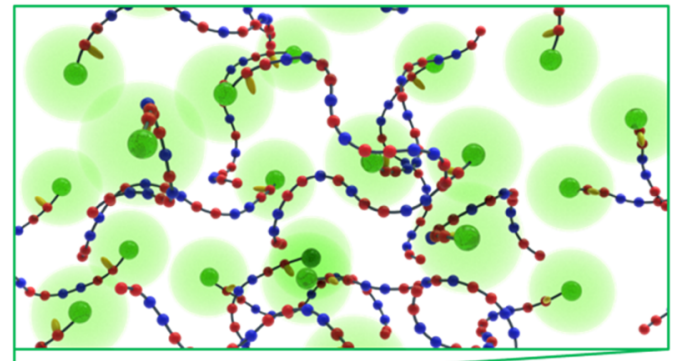

(b)

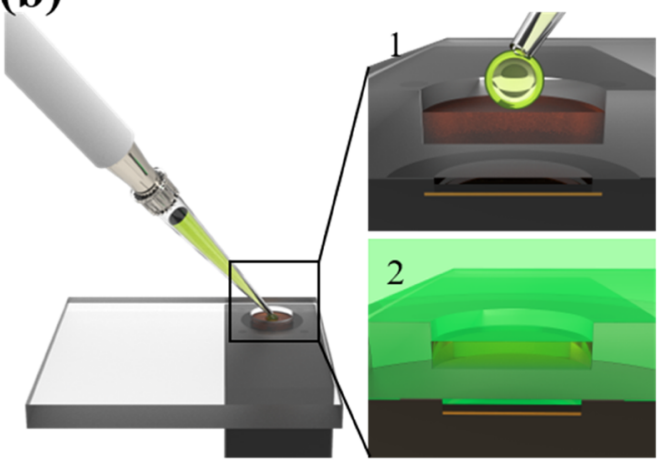

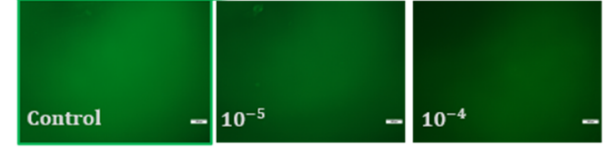

(d)

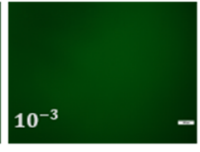

$10^{-2}$

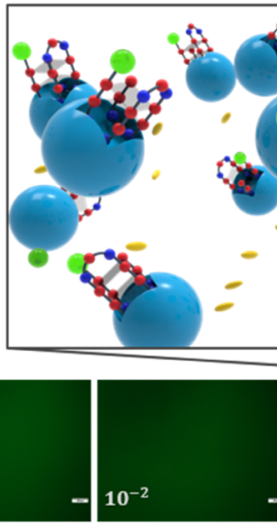

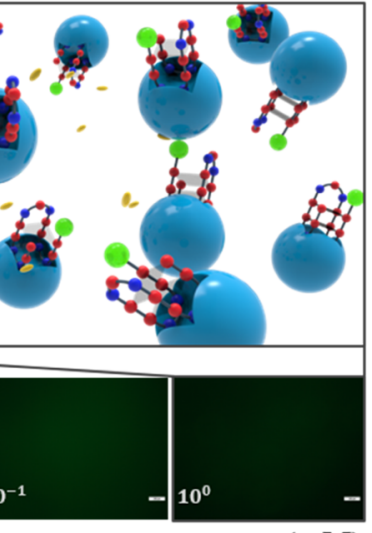

(mM)

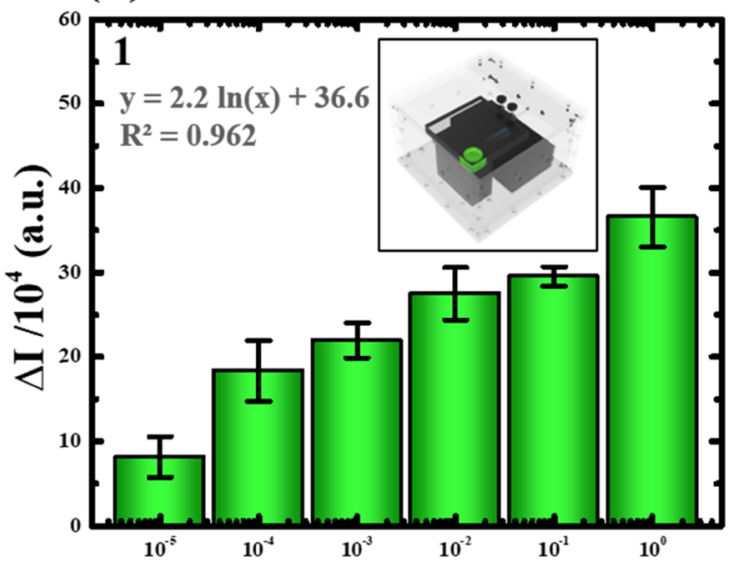

Thrombin concentration (mM)

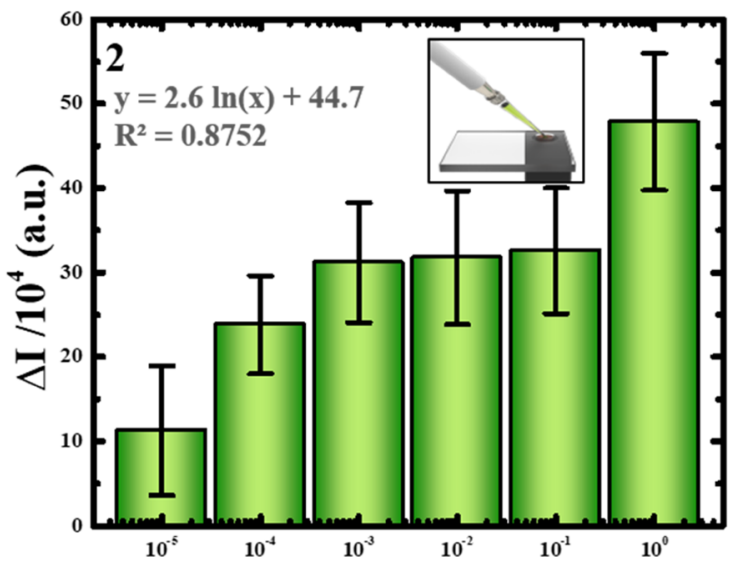

Thrombin concentration (mM)

Figure 4. Quantitative detection of the target thrombin based on two kinds of injection methods. (a) Injection of CL reagents into the reaction chamber through a microfluidic chip by air blowing. (b) Injection of the CL reagent into the reaction chamber via a micropipette. (c) CL optical images depend on the increase in the concentrations of thrombin from 0 to $1 \mathrm{mM}$. (d) Comparison of the variations in CL intensity $(\Delta I)$ according to the concentration of thrombin via the microfluidic chip and the micropipette. Error bar indicates the standard deviation of the CL intensity from five times in both injection methods.

\subsection{Detection Performance of the Microfluidic} Chip. Luminol was used to amplify the CL response and determine the ability of the microfluidic chip to quantitatively inject a given volume. The luminol reaction has been used in $\mathrm{H}_{2} \mathrm{O}_{2}$ determination, which is important for medical diagnosis because it is involved in many detection processes as an intermediate product. Figure 2a shows that luminol is oxidized in an alkaline solution in the presence of a catalyst $\left(\mathrm{CuSO}_{4}\right)$ to form 3-aminophthalate in an excited state, exhibiting wavelengths of maximum emission at $425 \mathrm{~nm}$. Figure $2 \mathrm{~b}$ illustrates that CL intensity is detected using the PMT sensor, which is located under the reaction chamber. The mixing solution of luminol-catalyst $\left(\mathrm{CuSO}_{4}\right)$ and $\mathrm{H}_{2} \mathrm{O}_{2}$ solution was filled into channels 1 and 2, respectively. Both solutions are carried in a series to the reaction chamber as well as $23 \mu \mathrm{L}$ by air injection. The luminol CL reaction occurs, and the luminescence intensity is immediately measured using the PMT. The relative $\mathrm{CL}$ intensity is sharply increased within a few seconds and dramatically decreased regardless of the concentrations of luminol, $\mathrm{CuSO}_{4}$, and $\mathrm{H}_{2} \mathrm{O}_{2}$ (Figure $2 \mathrm{c}$ ). Figure $2 \mathrm{~d}$ shows that 
the brightness of light emitted in the luminol CL reaction depends on the concentration of $\mathrm{H}_{2} \mathrm{O}_{2}$. The detection ability was also investigated on the basis of the increase in the concentrations of luminol, $\mathrm{CuSO}_{4}$, and $\mathrm{H}_{2} \mathrm{O}_{2}$. Figure $2 \mathrm{e}$ demonstrates that the quantitative $\mathrm{CL}$ response of $\mathrm{CuSO}_{4}$ with different concentrations while maintaining the concentration of the luminol and the oxidizer exhibits a dramatic increase with $0.1-0.5 \mathrm{mM}$ and was maintained regardless of the increase in concentration. For CL response of luminol with different concentrations while maintaining the concentration of the catalyst and the oxidizer exhibits a gradual increase with $0.1-$ $2.8 \mathrm{mM}$ and reaches the saturation intensity. The CL response of $\mathrm{H}_{2} \mathrm{O}_{2}$ with different concentrations while maintaining concentrations of luminol and catalyst exhibits a linear increase with 0.01-10 mM. This shows that the microfluidic chip had good sensitivity and reliability in the detection of $\mathrm{H}_{2} \mathrm{O}_{2}$, and the regression equation and correlation coefficient are indicated on the graph (Figures 2e and 3).

These results indicate that our microfluidic chip can detect the CL reaction with high sensitivity and control the reagent injection volume by blowing an appropriate amount of air without using any complex device.

2.3. Microchip-Based CL Sensor for Thrombin Detection Compared with a Micropipette System as an Application. The antithrombin aptamer is widely known to adopt a stable G-quadruplex structure when it binds to thrombin. $^{26-31}$ The conjugation of hemin into the resulting Gquadruplex TBA-thrombin complex produces an active TMPG that was used to catalyze the generation of CL. No fluorescence probes, quantum dots, and enzymes as amplifying labels were available, but the CL intensity emitted a relatively weaker dim light because of the absence of intra-CL resonance energy transfer (intra-CRET). ${ }^{32-34}$ Figure 3 illustrates the use of TBA labeled with a fluorescence probe (6-FAM)-thrombin complex for the generation of CL in the presence of TPA and $\mathrm{O}_{2}$. TMPG reacted with guanine of 6-FAM-TBA to emit dim light, which was generated from the reaction of TMPG and guanine of TBA can transfer energy to 6-FAM-TBA and to emit bright green light based on the principle of intra-CRET. The G-quadruplex TBA binds to thrombin and does not react with TMPG. The CL intensity of the TBA-thrombin complex decreases as the thrombin concentration increases. ${ }^{35-37}$ In recent studies on chemiluminescence immunoassay for biomarkers through the lab-on-a-chip are using the diffusion dominant mixing of the laminar flows in the microchannels. $^{38,39}$ This process needs a fluid injection system such as syringe pumps, which need to be controlled, and sample mountings. Indeed, microfluidic chemiluminescence immunoassay detection using a microfluidic chip and instrument has a complex process such as loading samples, incubation, and washing. It is necessary to prepare the complex microfluidic chip for loading reagents inside reservoirs and requires a lot of time. Also, before the stabilization of the flows, all of the sample fluids need to be wasted and precisely controlled. The ultrasensitive detection of thrombin was evaluated using the microfluidic chip, which was applied to all reagent solutions for precise injection without a complex device. In the experimental section, the detection ability of 6FAM-TBA for thrombin was compared with that of a micropipette and a microfluidic chip. First, various concentrations of the thrombin sample solution were mixed with 6FAM-TBA and incubated for $10 \mathrm{~min}$ at room temperature. Second, the reaction chamber was filled with a TMPG solution
$(220 \mu \mathrm{L})$ using a micropipette. Figure 4a shows that 6-FAMTBA-conjugated thrombin and TPA solution filled the channel volume and moved into the reaction chamber in a quantitative volume $(22 \mu \mathrm{L})$ through air blowing. The other injection method involving a micropipette can be used to simply drop the sample solution on the reaction chamber (Figure 4b). The guanines of free 6-FAM-TBA reacted with TMPG to emit light by intra-CRET, but thrombin arranged the G-quadruplex structure via the reduction in the binding site between TBA and TMPG. Figure $4 \mathrm{c}$ shows that the brightness of light emitted via the guanine CL reaction depended on the concentration of thrombin.

The performance of CL sensitivity was compared between the microfluidic chip and micropipette methods for detection in terms of linear detection range, reproducibility, and limit of detection (LOD). All of the injection volumes of the reagents in both methods were unified following the process. The performance of the two injection methods was verified by applying $220 \mu \mathrm{L}$ of TMPG and $22 \mu \mathrm{L}$ of target solution with thrombin at various concentrations. Figure $4 \mathrm{~d}$ shows that $\Delta I$ of the CL intensity linearly increased with thrombin concentration regardless of the target sample injection methods. In the case of a microfluidic chip, a linear relationship with a small error of variation was observed, and the LOD was $10^{-5} \mathrm{mM}$. The microfluidic chip also had good sensitivity and reliability in the detection of thrombin samples. The regression equation and correlation coefficient are shown inside the graphs. The regression values were comparable in both cases. However, the correlation coefficient and standard deviation of CL intensity differed depending on the sample injection methods. In the case of microfluidic chips, the variation in the standard deviation was constant even though several microfluidic chips were used. A large variation in the standard deviation was found when a micropipette was utilized. Therefore, CL detection using the microfluidic chip platform could lessen errors in manual injection and precisely control the reagent volumes without requiring complex devices, which promised more quantitative analysis of CL.

\section{CONCLUSIONS}

In this study, a portable microfluidic CL analysis platform is developed to quantitatively control reagent solutions. The channel size and height of the microfluidic chip can be designed with the exact sample volume to be transferred to a reaction chamber. Air blowing by syringe without any complex device is applied to control the reagent volume. The microfluidic platform can be used to detect luminol, $\mathrm{CuSO}_{4}$, and clinical targets, such as thrombin, which has an important role in some blood-related diseases. Specifically, the early mixing of CL reagents is solved using this microfluidic chip platform, which is composed of four ports, two channels, and a reaction or view chamber for the detection of CL intensity. These results indicate that the microfluidic chip platform improves the reproducibility of CL intensity to detect thrombin and exhibits a broad linear range of $1.0 \times 10^{-5}$ to $1 \mathrm{mM}$. The CL signal variations caused by the controlled reagent volume can be eliminated with this platform. Therefore, the proposed CL sensor based on a microfluidic chip may be utilized to detect thrombin in actual samples. However, its application to samples with a large volume, such as several thousand microliters, is limited because of difficulty in extending the size and height of channels, which are 
(a)

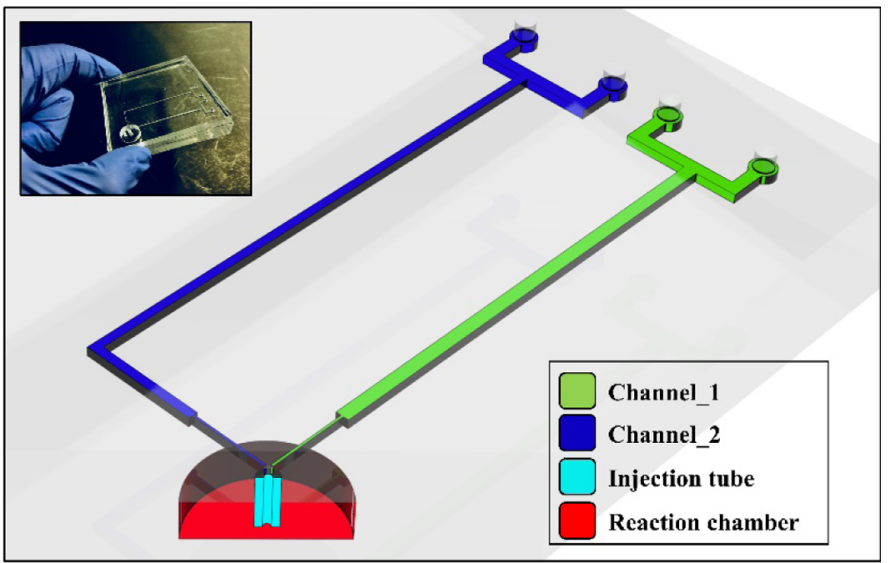

(b)

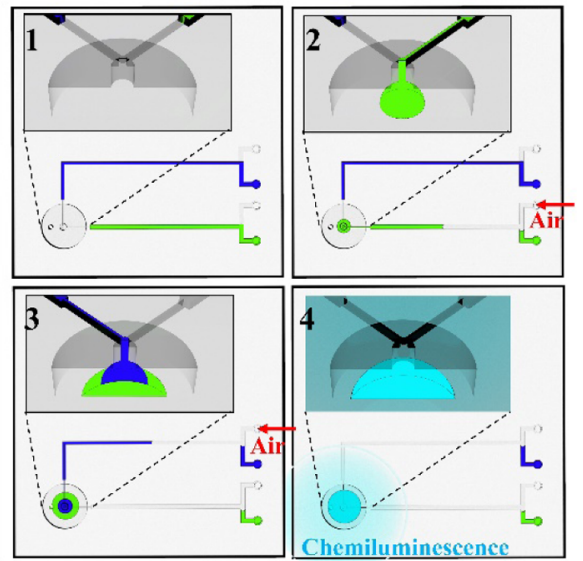

(c)
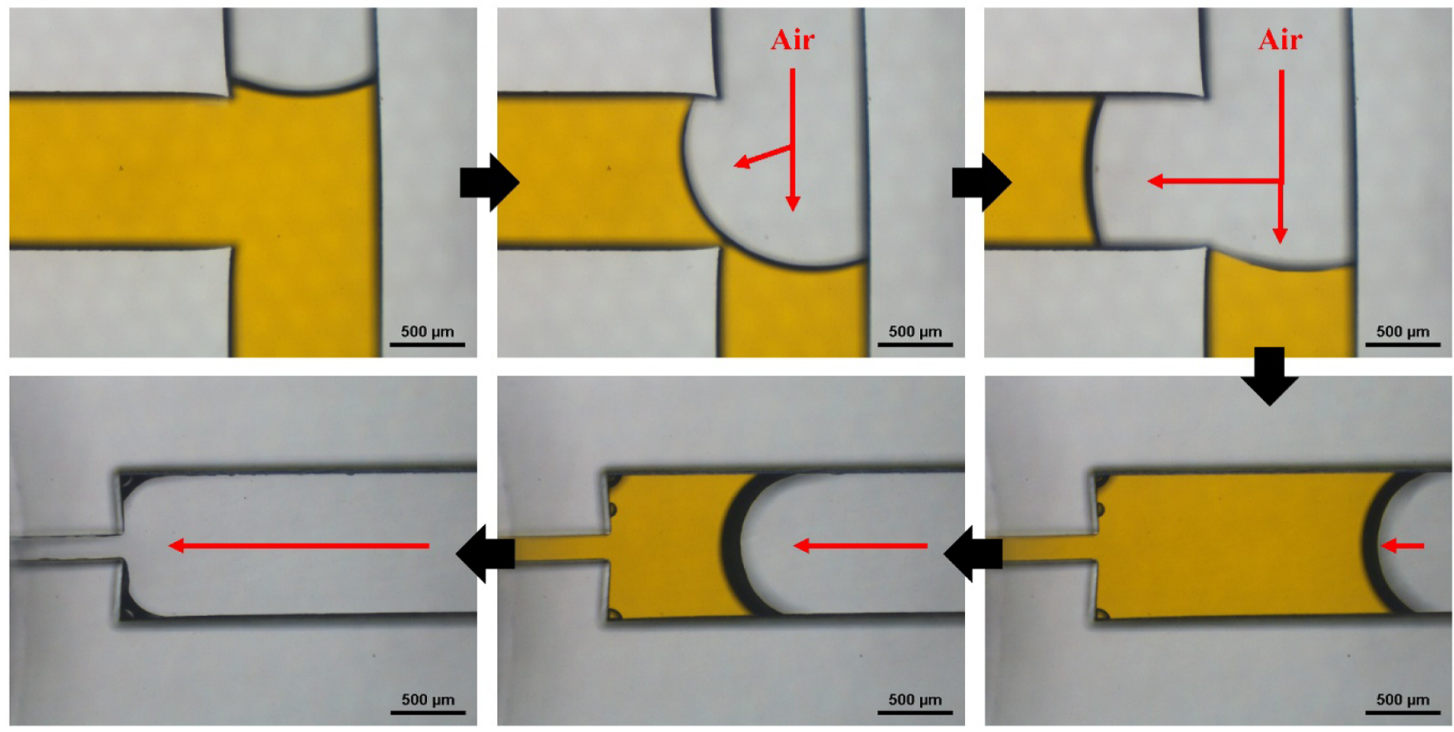

Figure 5. (a) Photo image and illustration of the fabricated microfluidic chip composed of two channels connected to four injection ports, an injection tube, and reaction chamber parts. (b) Schematic of the reagent solution injection from the channels to the reaction chamber quantitatively. (1) Microchannels were completely filled with each reagent. $(2,3)$ Two reagents from the channels were quantitatively injected into the reaction chamber by airflow through another injection port. (4) CL intensity was detected in the reaction chamber via the chemical reaction of the sample reagents. (c) Photo images of the process of a quantitative injection reagent in channels. The microchannels were fully filled with the reagent solution that was moved into the reaction chamber via air blowing from an empty inlet port. The reagent solution was separated at the cross section of the channel and reached an amount of quantitative volume into the reaction chamber. (d) Signal of CL intensity immediately increased in two reagents mixed in the reaction chamber compared with that in the control sample of pure water without any reaction reagents.

fabricated via MEMS. Nevertheless, it can be quantitatively applied to an intra-CRET process as a readout CL signal.

\section{EXPERIMENTAL SECTION}

4.1. Materials and Regents. Thrombin aptamer (TBA) and TBA conjugated with 6-carboxyfluorescein (6-FAM) were purchased from Geno Tech. Corp. (Daejeon, South Korea). 6 FAM-TBA was HPLC purified and freeze-dried by the supplier and used as provided and diluted in $10 \mathrm{mM}$ phosphate buffer solution with $\mathrm{pH} 7.5$ to prepare $100 \mu \mathrm{M}$ stock solutions. The following ssDNA sequence was used: 5'-6-FAMTTTGGTTGGTGTGGTTGG-3'. Tetra- $n$-propylammonium hydroxide (TPA, 40\% w/w aqueous solution) and 3-aminophthalhydrazide (luminol) were procured from Alfa Aesar. Human thrombin was bought from Heamatologic Tech. Incorp (HCT-0020). Copper(II) sulfate pentahydrate $\left(\mathrm{CuSO}_{4}\right)$. All other reagents were obtained from commercial suppliers (Sigma-Aldrich) and used without further purification. All solvents used were of analytical reagent grade. 3,4,5Trimethoxylphenyl-glyoxal (TMPG) was synthesized under the following previously described conditions. ${ }^{40}$ 3,4,5-Trimethoxyacetophenone $(10.5 \mathrm{~g}, 50 \mathrm{mmol})$ was added to a stirred solution of selenium dioxide $(5.0 \mathrm{~g}, 45 \mathrm{mmol})$ in dioxane (40 $\mathrm{mL}$ ) at $40{ }^{\circ} \mathrm{C}$. The mixture was refluxed for $2 \mathrm{~h}$, and selenium dioxide $(5.0 \mathrm{~g}, 45 \mathrm{mmol})$ was added to the reaction mixture. After being refluxed for $3 \mathrm{~h}$, the mixture was filtered to remove insoluble selenium. The filtrate was mixed with $240 \mathrm{~mL}$ of distilled water and kept at $4{ }^{\circ} \mathrm{C}$ for approximately $15 \mathrm{~h}$. The formed precipitate was recrystallized from water to produce pure $\mathrm{TMPG}$ as a colorless needle $\left(\mathrm{mp} 101-102{ }^{\circ} \mathrm{C}\right.$, yield $=$ $60-70 \%)$. TMPG is unstable in solvents, so it was prepared just before use. A $1.0 \mathrm{M}$ stock solution of 3-aminophthalhydrazide (luminol) was prepared by dissolving luminol in $100 \mathrm{mM}$ sodium hydroxide in $10 \mathrm{mM}$ phosphate buffer 
solution. An oxidant solution of hydrogen peroxide $\left(\mathrm{H}_{2} \mathrm{O}_{2}\right)$ was prepared by diluting $30 \%(\mathrm{v} / \mathrm{v}) \mathrm{H}_{2} \mathrm{O}_{2}$ stock solution.

4.2. Design and Fabrication of a Microfluidic Chip Platform. Microfluidic chips $(60 \mathrm{~mm} \times 60 \mathrm{~mm} \times 8 \mathrm{~mm})$ were fabricated via PDMS casting using a positive silicon wafer and an aluminum mold. The fabricated chip was composed of three layers: a top microfluidic layer, a middle layer with tubing holes, and a bottom substrate layer with a reaction and measurement chamber. The detailed process is described in Figure S1. The top microfluidic layer is composed of two microchannels that can quantitatively classify and inject reagent and target solutions. The microfluidic chip also had a chamber with $10 \mathrm{~mm}$ diameter and $3 \mathrm{~mm}$ height for the mixing of injected reagents and transmitting chemical luminescent signals from the mixture to a photomultiplier tube (PMT) sensor. The microchannels and the chamber were hierarchically arranged, and the structure was bound with three PDMS layers. Afterward, a $130 \mathrm{~mm} \times 130 \mathrm{~mm} \times 120 \mathrm{~mm}$ chemical luminometric device containing a microfluidic chip controller, a PMT photosensor module, and a darkroom for blocking external light sources was set up to measure chemical luminescent reactions inside the fabricated microfluidic chips.

4.3. Detection of Luminol and Thrombin by CL. For luminol analysis, a $\mathrm{CL}$ assay was performed in a microfluidic chip containing $1.3 \mathrm{M} \mathrm{H}_{2} \mathrm{O}_{2}$ and different concentrations of a luminol solution and a $\mathrm{CuSO}_{4}$ solution as a catalyst at channels 1 and 2, respectively. Different concentrations of thrombin were added to $100 \mathrm{nM} 6$-FAM-TBA prepared in water at room temperature for $10 \mathrm{~min}$ to detect thrombin. Then, $2 \mathrm{mM}$ TMPG and $10 \mathrm{mM}$ TPA were prepared in $N, N$-dimethylformamide (DMF) and water, respectively. The solution of 6FAM-TBA-thrombin $(11 \mu \mathrm{L})$ and TPA $(11 \mu \mathrm{L})$ was poured into the microfluidic channel, and $8 \mathrm{mM} \mathrm{TMPG}(220 \mu \mathrm{L})$ was added dropwise to the reaction chamber via a micropipette. The light emission intensity from the reaction chamber was measured immediately.

The portable chemical luminometric device was approximately $130 \mathrm{~mm} \times 130 \mathrm{~mm} \times 120 \mathrm{~mm}$ and had two floors composed of (1) a system of fluid transportation in microchannels to assay the chamber and (2) a detector of CL intensity via PMT sensor modules. The bottom floor had a PMT sensor module consisting of a photosensor module with PMT, a voltage controller, and a power supply (H10722-110, HAMAMATSU). The top floor had a darkroom for installing the microfluidic chip and blocking external light sources and pistons to control syringe pressure (Figure S2). The inside of the device blocks external light sources to preserve the darkroom, and a weak CL intensity could be sensitively detected. The bottom floor had a microfluidic chip hold (60 $\mathrm{mm} \times 60 \mathrm{~mm} \times 4 \mathrm{~mm})$ and a $10 \mathrm{~mm}$ diameter of the view hole to deliver a photon signal to the PMT photosensor. The sensor was connected to an oscilloscope (Picoscope 4262, Picotech) and a computer to analyze the changes in the measured CL intensity. Exclusive software provided by a manufacturing company was used.

4.4. Precise Control Injection of Reagents. Channel structures were designed to control the injected reagent solution from two channels to the reaction chamber. The microfluidic chip was composed of two channels, four inlet ports, and an injection tube connected to the reaction chamber (Figure 5a). Briefly, each channel was filled with reagent solutions through one of the ports until the injection tube was reached, and adequate air volume was sequentially injected via the other inlet ports (Figure 5b). In the cross section between the channel and the inlet line, the precise volume of the reagent solution was moved to the reaction chamber, and an extra solution was retained in another port area. The volume of the reagent solutions was precisely controlled depending on the width, length, and height of the channels. In Figure 5c, the quantitative injection reagent is moving on microfluidic channel following by above process. The filled-up reagent solution was moved into the reaction chamber by air blowing from an empty inlet port and separated at the cross section of the channel. The reagent finally reached an amount of quantitative volume into the reaction chamber. Figure 5d shows the CL intensity when two reagent solutions reached the reaction chamber, immediately increasing within a few seconds. Conversely, the signal did not increase in the pure water solution as the control sample. Figure 5c presents the photo images of the quantitative injection of the reagent into the channels. The microchannels were fully filled with the reagent solution, which was then moved into the reaction chamber by air blowing from an empty inlet port. The reagent solution was separated at the cross section of the channel and reached an amount of quantitative volume into the reaction chamber.

\section{ASSOCIATED CONTENT}

\section{Supporting Information}

The Supporting Information is available free of charge at https://pubs.acs.org/doi/10.1021/acsomega.1c03347.

Details of the fabrication methods of microfluidic chip and setup of the device (PDF)

\section{AUTHOR INFORMATION}

\section{Corresponding Authors}

Ho-Sup Jung - Center for Food and Bioconvergence, Department of Food Science and Biotechnology, Seoul National University, Seoul 08826, South Korea; ○ orcid.org/0000-0001-5991-2256; Email: jhs@snu.ac.kr

Moon Kyu Kwak - School of Mechanical Engineering, Kyungpook National University, Daegu 41566, South Korea; 이이.org/0000-0002-8902-7685; Email: mkkwak@ knu.ac.kr

\section{Authors}

Bongsu Kang - School of Mechanical Engineering, Kyungpook National University, Daegu 41566, South Korea

Sunghak Choi - Center for Food and Bioconvergence, Department of Food Science and Biotechnology, Seoul National University, Seoul 08826, South Korea

Keesung Kim - Research Inst. of Advanced Materials, College of Engineering, Seoul National University, Seoul 08826, South Korea

Complete contact information is available at:

https://pubs.acs.org/10.1021/acsomega.1c03347

\section{Author Contributions}

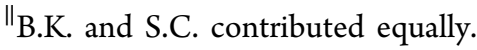

\section{Notes}

The authors declare no competing financial interest.

\section{ACKNOWLEDGMENTS}

This work was supported by the National Research Foundation of Korea grant (NRF-2020R1A4A1018652 and 
2019R1A2C1086766), the Basic Research Program funded by the Korea Institute of Machinery and Materials (grant number NK226E), the Cooperative Research Program for Agriculture Science and Technology Development (Project No. PJ01574703), and the Rural Development Administration, Republic of Korea and Korea Institute of Planning and Evaluation for Technology in Food, Agriculture and Forestry (IPET) through Agro and Livestock Products Safety·Flow Management Technology Development Program, funded by the Ministry of Agriculture, Food and Rural Affairs (MAFRA) (118105-3).

\section{REFERENCES}

(1) Gubala, V.; Harris, L. F.; Ricco, A. J.; Tan, M. X.; Williams, D. E. Point of care diagnostics: status and future. Anal. Chem. 2012, 84, $487-515$.

(2) Peeling, R.; Mabey, D. Point-of-care tests for diagnosing infections in the developing world. Clin. Microbiol. Infect. 2010, 16, $1062-1069$.

(3) Song, Y.; Huang, Y.-Y.; Liu, X.; Zhang, X.; Ferrari, M.; Qin, L. Point-of-care technologies for molecular diagnostics using a drop of blood. Trends Biotechnol. 2014, 32, 132-139.

(4) Ballerini, D. R.; Li, X.; Shen, W. Patterned paper and alternative materials as substrates for low-cost microfluidic diagnostics. Microfluid. Nanofluid. 2012, 13, 769-787.

(5) Yetisen, A. K.; Akram, M. S.; Lowe, C. R. Paper-based microfluidic point-of-care diagnostic devices. Lab Chip 2013, 13, 2210-2251.

(6) Van Dam, R. M. Solvent-Resistant Elastomeric Microfluidic Devices and Applications. Dissertations \& Theses, California Institute of Technology, 2006

(7) Volpatti, L. R.; Yetisen, A. K. Commercialization of microfluidic devices. Trends Biotechnol. 2014, 32, 347-350.

(8) Terry, S. C.; Jerman, J. H.; Angell, J. B. A gas chromatographic air analyzer fabricated on a silicon wafer. IEEE Trans. Electron Devices 1979, 26, 1880-1886.

(9) Lorenz, H.; Despont, M.; Fahrni, N.; Brugger, J.; Vettiger, P.; Renaud, P. High-aspect-ratio, ultrathick, negative-tone near-UV photoresist and its applications for MEMS. Sens. Actuators, A 1998, $64,33-39$.

(10) del Campo, A.; Greiner, C. SU-8: a photoresist for high-aspectratio and 3D submicron lithography. J. Micromech. Microeng. 2007, 17, R81.

(11) Xia, Y.; Whitesides, G. M. Soft lithography. Annu. Rev. Mater. Sci. 1998, 28, 153-184.

(12) Qin, D.; Xia, Y.; Whitesides, G. M. Soft lithography for microand nanoscale patterning. Nat. Protoc. 2010, 5, 491-502.

(13) Srinivasan, V.; Pamula, V. K.; Fair, R. B. An integrated digital microfluidic lab-on-a-chip for clinical diagnostics on human physiological fluids. Lab Chip 2004, 4, 310-315.

(14) Thorsen, T.; Maerkl, S. J.; Quake, S. R. Microfluidic large-scale integration. Science 2002, 298, 580-584.

(15) Myers, F. B.; Lee, L. P. Innovations in optical microfluidic technologies for point-of-care diagnostics. Lab Chip 2008, 8, 20152031.

(16) Wang, M. M.; Tu, E.; Raymond, D. E.; Yang, J. M.; Zhang, H.; Hagen, N.; Dees, B.; Mercer, E. M.; Forster, A. H.; Kariv, I.; et al. Microfluidic sorting of mammalian cells by optical force switching. Nat. Biotechnol. 2005, 23, 83-87.

(17) Juris, A.; Balzani, V.; Barigelletti, F.; Campagna, S.; Belser, Pl.; von Zelewsky, Av. Ru (II) polypyridine complexes: photophysics, photochemistry, eletrochemistry, and chemiluminescence. Coord. Chem. Rev. 1988, 84, 85-277.

(18) DeChatelet, L.; Long, G.; Shirley, P.; Bass, D.; Thomas, M.; Henderson, F.; Cohen, M. Mechanism of the luminol-dependent chemiluminescence of human neutrophils. J. Immunol. 1982, 129, $1589-1593$.
(19) Divi, R. L.; Beland, F. A.; Fu, P. P.; Von Tungeln, L. S.; Schoket, B.; Camara, J. E.; Ghei, M.; Rothman, N.; Sinha, R.; Poirier, M. C. Highly sensitive chemiluminescence immunoassay for benzo [a] pyrene-DNA adducts: validation by comparison with other methods, and use in human biomonitoring. Carcinogenesis 2002, 23, 2043-2049.

(20) Fujii, Y.; Henares, T. G.; Kawamura, K.; Endo, T.; Hisamoto, H. Bulk-and surface-modified combinable PDMS capillary sensor array as an easy-to-use sensing device with enhanced sensitivity to elevated concentrations of multiple serum sample components. Lab Chip 2012, 12, 1522-1526.

(21) Sarveswaran, K.; Kurz, V.; Dong, Z.; Tanaka, T.; Penny, S.; Timp, G. Synthetic capillaries to control microscopic blood flow. Sci. Rep. 2016, 6, No. 21885.

(22) Chow, A. W. Lab-on-Chip: Opportunities for chemical engineering. American Institute of Chemical Engineers. AIChE J. 2002, 48, 1590.

(23) DeWitt, S. H. Microreactors for chemical synthesis. Curr. Opin. Chem. Biol. 1999, 3, 350-356.

(24) Honrado, C.; Dong, T. Development and optimization of an integrated capillary-based opto-microfluidic device for chemiluminescence quantitative detection. J. Micromech. Microeng. 2014, 24, No. 125023.

(25) Bostick, D. T.; Hercules, D. M. Quantitative determination of blood glucose using enzyme induced chemiluminescence of luminol. Anal. Chem. 1975, 47, 447-452.

(26) Cho, S.; Park, L.; Chong, R.; Kim, Y. T.; Lee, J. H. Rapid and simple G-quadruplex DNA aptasensor with guanine chemiluminescence detection. Biosens. Bioelectron. 2014, 52, 310-316.

(27) Deng, B.; Lin, Y.; Wang, C.; Li, F.; Wang, Z.; Zhang, H.; Li, X.F.; Le, X. C. Aptamer binding assays for proteins: the thrombin example-a review. Anal. Chim. Acta 2014, 837, 1-15.

(28) Li, J. J.; Fang, X.; Tan, W. Molecular aptamer beacons for realtime protein recognition. Biochem. Biophys. Res. Commun. 2002, 292, $31-40$.

(29) Hamaguchi, N.; Ellington, A.; Stanton, M. Aptamer beacons for the direct detection of proteins. Anal. Biochem. 2001, 294, 126-131.

(30) Aaldering, L. J.; Poongavanam, V.; Langkjær, N.; Murugan, N. A.; Jørgensen, P. T.; Wengel, J.; Veedu, R. N. Development of an Efficient G-Quadruplex-Stabilised Thrombin-Binding Aptamer Containing a Three-Carbon Spacer Molecule. ChemBioChem 2017, 18, $755-763$.

(31) Russo Krauss, I.; Merlino, A.; Giancola, C.; Randazzo, A.; Mazzarella, L.; Sica, F. Thrombin-aptamer recognition: a revealed ambiguity. Nucleic Acids Res. 2011, 39, 7858-7867.

(32) Yamasuji, M.; Shibata, T.; Kabashima, T.; Kai, M. Chemiluminescence detection of telomere DNA in human cells on a membrane by using fluorescein-5-isothiocyanate-labeled primers. Anal. Biochem. 2011, 413, 50-54.

(33) Yan, Y.; Wang, X.-y.; Hai, X.; Song, W.; Ding, C.; Cao, J.; Bi, S. Chemiluminescence resonance energy transfer: From mechanisms to analytical applications. TrAC, Trends Anal. Chem. 2020, 123, No. 115755.

(34) Chong, J.; Chong, H.; Lee, J. H. A chemiluminescent dualaptasensor capable of simultaneously quantifying prostate specific antigen and vascular endothelial growth factor. Anal. Biochem. 2019, 564-565, 102-107.

(35) Kai, M.; Ohkura, Y.; Yonekura, S.; Iwasaki, M. Chemiluminescence determination of guanine and its nucleosides and nucleotides using phenylglyoxal. Anal. Chim. Acta 1994, 287, 75-81.

(36) Shapiro, R.; Cohen, B. I.; Shiuey, S.-J.; Maurer, H. Reaction of guanine with glyoxal, pyruvaldehyde, and kethoxal, and the structure of the acylguanines. Synthesis of N2-alkylguanines. Biochemistry 1969, $8,238-245$.

(37) Mitchell, D.; Ritchey, L. E.; Park, H.; Babitzke, P.; Assmann, S. M.; Bevilacqua, P. C. Glyoxals as in vivo RNA structural probes of guanine base-pairing. RNA 2018, 24, 114-124.

(38) Lin, Y.; Li, J.; Wang, Y.; Sun, Y.; Ding, C.; Sun, W.; Luo, C. A chemiluminescence biosensor for the detection of thrombin based on 
the aptamer composites. Spectrochim. Acta, Part A 2018, 192, 153158.

(39) Le, N. C. H.; Gel, M.; Zhu, Y.; Wang, J.; Dacres, H.; Anderson, A.; Trowell, S. C. Sub-nanomolar detection of thrombin activity on a microfluidic chip. Biomicrofluidics 2014, 8, No. 064110.

(40) Kai, M.; Kishida, S.; Sakai, K. A chemiluminescence derivatization method for detecting nucleic acids and DNA probes using a trimethoxyphenylglyoxal reagent that recognizes guanine. Anal. Chim. Acta 1999, 381, 155-163. 